\title{
Stem cell therapy facilitates tolerance in renal transplantation
}

Background: Strategies for tolerance induction (TI) include induction of chimerism and/or clonal deletion. We present 5 -year experience of TI in living-donor related renal transplantation (LDRT) using adipose derived mesenchymal stem cell (AD-MSC) and hematopoietic SC (HSC) infusion with supportive therapy.

Methods: Twenty patients divided in 2 equal, demographically balanced groups underwent LDRT under TI protocol (TIP) consisting of conditioning with Bortezomib, Methyl prednisone, rabbit-anti-thymoglobulin and Rituximab. Group-1 were administered in-vitro generated AD-MSC and HSC, group-2 were administered donor specific transfusion. Transplantation was carried out with acceptable lymphocyte cross-match, $T$ and B-cell flow cross-match, single antigen assay and negative mixed lymphocyte reaction (MLR). No conventional immunosuppression was to be administered. Monitoring included serum creatinine ( $\mathrm{SCr}-\mathrm{mg} / \mathrm{dL}$ ), donor specific antibodies (DSA) and MLR. Protocol biopsies were planned after 100 days and yearly in willing patients. In event of rejection/ DSA/ MLR, rescue immunosuppression was planned.

Results: Over mean 5.4 (range: 4.9-6.3) year follow-up patient survival was $80 \%$ in group-1 and $90 \%$ in group-2; deathcensored graft survival was $90 \%$ in group- 1 and $70 \%$ in group-2. Mean SCr was 1.52 in group-1, and 1.97 in group-2. Five patients from group-1 and 3 from group-2 were on no conventional immunosuppression, 2 patients of group- 1 and 1 of group-2 were on two immunosuppressants and 1 patient of group- 2 was on 3 immunosuppressants. DSA appeared in 2 patients of group-1 without affecting graft function and 4 of group-2 causing graft dysfunction. MLR was negative in both groups.

Conclusion: SCs facilitate TI in LDRT under non-myeloablative conditioning.

KEYWORDS: Mesenchymal stem cells, hematopoietic stem cells, renal transplantation, tolerance, nonmyeloablative conditioning, clonal deletion

\section{Abbreviations}

ABMR: Antibody Mediated Rejections; ADMSC: Adipose Tissue Derived Mesenchymal Stem Cells; AHG: Anti-Human Globulin; ASHI: American Society of Histocompatibility and Immunogenetics; CGN: Chronic Glomerulonephritis; CNI: Calcineurin Inhibitor; CRF: Chronic Renal Failure; DMEM: Dulbecco's Modified Eagle's Medium; DSA: Donor Specific Antibody; DST: Donor Specific Transfusion; FCM: Flow Cross-Match; FITC: Fluorescent Isothiocynate; GVHD: Graft Versus Host Disease; HSC: Hematopoietic Stem Cells; HSCT: Hematopoietic Stem Cell Transplantation; IF/TA: Interstitial Fibrosis/ Tubular Atrophy; LA: Local Anesthesia; LCM: Lymphocyte Cross-Match; LDRT: Living Donor Renal Transplantation; MCS: Median Channel Shift; MFI: Mean Fluorescent Intensity; MLR: Mixed Lymphocyte Reaction;
MSC: Mesenchymal Stem Cells; PBSC: Peripheral Blood Stem Cell; PE: Phycoerythrin; r-ATG: Rabbit Antithymoglobulin; RT: Renal Transplantation; SA: Single Antigen; SCT: Stem Cell Therapy; SCr: Serum Creatinine; TCR: T-cell Mediated Rejection; TI: Tolerance Induction; TIP: Tolerance Induction Protocol.

\section{Background}

Transplantation tolerance can be defined as survival of an allograft in the recipient on no immunosuppressive treatment without any rejection episodes [1]. However, there is no time-line drawn in this definition. In humans, new term of "operational tolerance" evolved where the grafted organ survived successfully for $\geq 1$-year after weaning off immunosuppression $[2,3]$. Pathways of tolerance are believed to be central involving thymic clonal deletion or peripheral involving deletion of alloreactive

\section{Aruna V Vanikar ${ }^{* 1}$ \&} Hargovind L Trivedi ${ }^{2}$

${ }^{1}$ Department of Pathology, Lab Medicine, Transfusion Services \& Immunohematology, and Department of Regenerative Medicine and Cell Therapy, G.R. Doshi and K.M. Mehta Institute of Kidney Diseases and Research Centre and Dr. H.L. Trivedi Institute of Transplantation Sciences (IKDRC-ITS), Civil hospital campus,

Asarwa, Ahmedabad, India

${ }^{2}$ Department of Nephrology and Transplantation Medicine, and Department of Regenerative Medicine and Stem Cell Therapy, G.R. Doshi and K.M. Mehta Institute of Kidney Diseases and Research Centre and Dr. H.L. Trivedi Institute of Transplantation Sciences (IKDRC-ITS), Civil hospital campus, Asarwa, Ahmedabad, India

*Author for correspondence: vanikararuna@yahoo.com 
T-cells in the periphery.4 Mixed chimerism with donor hematopoietic stem cells (HSC) was believed to be essential for tolerance induction (TI) [4-6]. The role of persistence of donor chimerism remains questionable since it has been documented that following initial engraftment supported by transient induction of donor chimerism, later on, durable engraftment and persistence of low frequency of host-vs-graft cytotoxic T-cell precursor cells can be maintained by persistence of donor alloantigens provided by the allograft itself even when chimerism is lost [7]. Gradual withdrawal of immunosuppression has remained the cornerstone for induction of transplantation tolerance in all studies described so far in literature. Mesenchymal stem cells (MSC) have come-up as the key players in immunomodulation in the last decade [8]. We describe results of a unique observational study aiming for TI in recipients of living donor related renal transplantation (LDRT) using no conventional immunosuppression (other than low dose Prednisone), using donor adipose tissue derived MSC (AD-MSC) generated by our own technique [9].

\section{Methods}

Patients with established chronic renal failure (CRF) with related blood-group matching donors were included in the study after explaining in detail all the procedures, risks, and possible benefits. Donors/ patients with $\mathrm{HIV} / \mathrm{HBsAg} / \mathrm{HCV}$ positivity, or any comorbid conditions were excluded.

\section{- Study design and treatment protocols:}

This was a prospective open-labeled twoarmed observational clinical study of patients transplanted between December 09 and April 11 with 10 patients in each arm, for LDRT using a self-designed TIP. TI protocol (TIP) and informed consent forms were approved by Institutional Review Board (IKDRCITS- CDB/ MCDB-09-2009).

\section{- Patient-donor demographics:}

Group- 1 included 7 males and 3 females with mean age of $31.6 \pm 5.8$ years. Original disease causing CRF was chronic glomerulonephritis (CGN) in 7, benign nephrosclerosis in 2 and lupus nephritis in 1 patient. They had received mean $2.5 \pm 3.37$ third party blood transfusions and $27.1 \pm 5.3$ dialysis. Donors were parents in 5 , spouses in 4 and sibling in 1 patient. There were 6 female to male transplants, 3 male to female and 1 male-to-male transplant. Mean donor age was $43.6 \pm 11.9$ years with mean HLA-match of $2.33 \pm 1.4$.

In group-2 all patients were males with mean age of $33.7 \pm 8.9$ years. Original disease causing CRF was CGN in 7, benign nephrosclerosis in 2 and chronic tubulointerstitial nephritis in 1 patient. They had received mean $1.4 \pm$ 2.17 third party transfusions and $26.1 \pm 11.49$ dialysis. Donors were parents in 5 and spouses in 5 patients. All donors were females. Mean donor age was $42.5 \pm 11.3$ years with mean HLA-match of $2.4 \pm 1.35$. There was no statistically significant difference in patient-donor demographics of the 2 groups (TABLE 1).

Group-1 were administered a new combination of stem cell therapy (SCT) and group-2 were administered $60 \mathrm{ml}$ donor specific transfusions (DST), while keeping same remaining steps. SC were generated from corresponding kidney donors.

\section{- Conditioning strategies:}

TIP used for both groups are mentioned in FIGURE 1A and 1B. TIP for group-1 consisted

\begin{tabular}{|c|c|c|c|}
\hline DEMOGRAPHICS & Group-1 $(n=10)(S C T)$ & Group-2 $(n=10)(D S T)$ & P-value \\
\hline Male: female & $7: 3$ & $10: 0$ & 0.060 \\
\hline Age in years (recipient) & $31.6 \pm 5.8$ & $33.7 \pm 8.9$ & 0.539 \\
\hline Age in years (donor) & $43.6 \pm 11.9$ & $42.5 \pm 11.3$ & 0.833 \\
\hline Donor recipient HLA match & $2.33 \pm 1.4$ & $2.4 \pm 1.35$ & 0.911 \\
\hline Mean third party transfusions & $2.5 \pm 3.37$ & $1.4 \pm 2.17$ & 0.397 \\
\hline Mean hemodialysis procedures & $27.1 \pm 5.32$ & $26 \pm 11.49$ & 0.787 \\
\hline \multicolumn{4}{|l|}{ DONOR RELATION } \\
\hline Parents & $5(50 \%)$ & $5(50 \%)$ & - \\
\hline Spousal & $4(40 \%)$ & $5(50 \%)$ & 0.653 \\
\hline Siblings & $1(10 \%)$ & 0 & 0.305 \\
\hline \multicolumn{4}{|l|}{ ORIGINAL DISEASE } \\
\hline Chronic Glomerulonephritis & $7(70 \%)$ & $7(70 \%)$ & - \\
\hline Nephrosclerosis & $2(20 \%)$ & $2(20 \%)$ & - \\
\hline Lupus nephritis & $1(10 \%)$ & 0 & 0.305 \\
\hline Chronic tubule-interstitial nephritis & 0 & $1(10 \%)$ & 0.305 \\
\hline
\end{tabular}




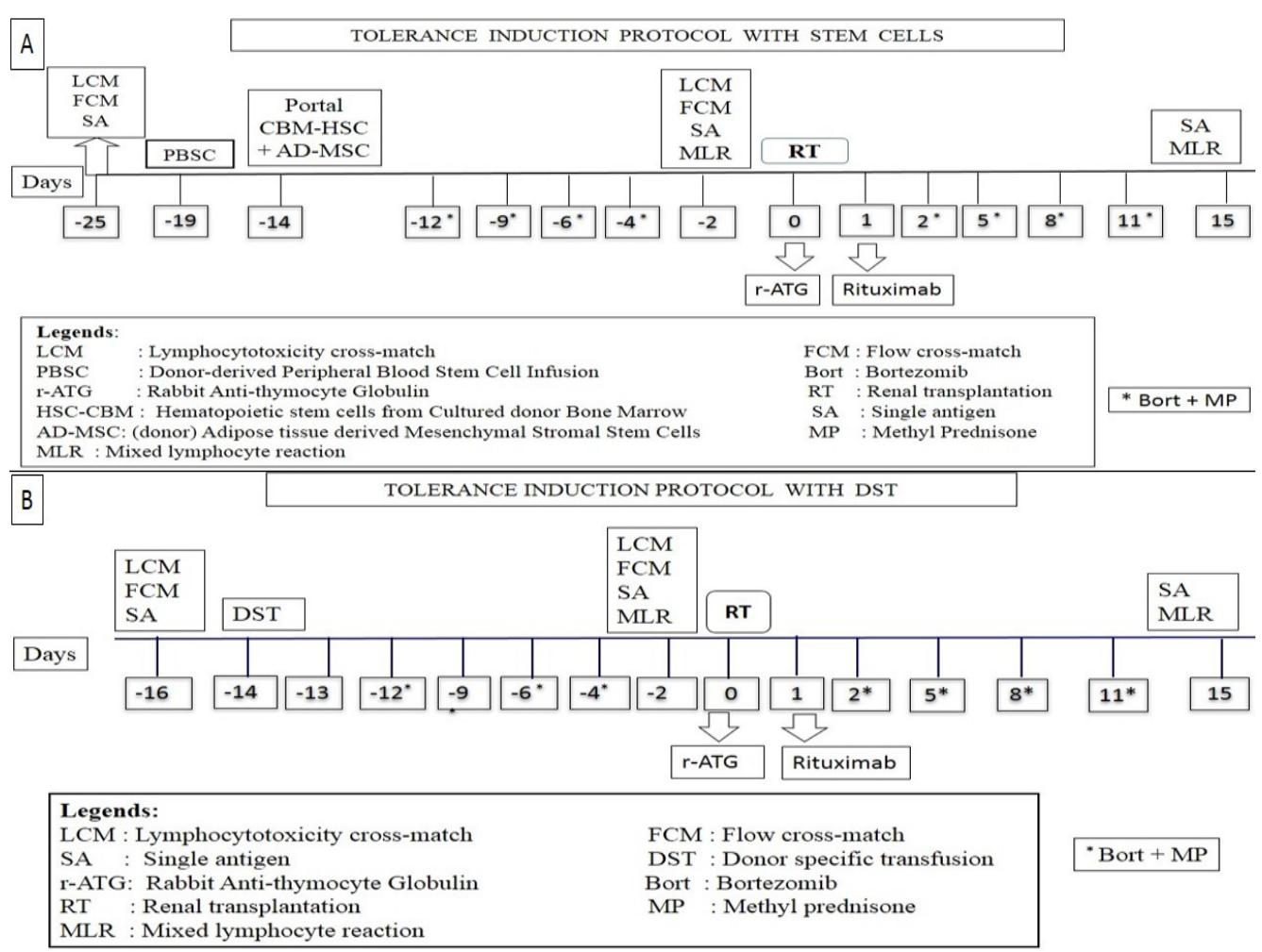

FIGURE 1 On upper quadrant Figure A, depicting tolerance induction protocol using stem cells for group-1, on lower quadrant Figure B depicting tolerance induction protocol using donor specific transfusion for group-2

of baseline lymphocyte cross-match (LCM), flow cross-match (FCM) and single antigen (SA) testing for donor specific antibodies (DSA) on day -25. Peripheral blood stem cell (PBSC) infusion on day -19 , and portal infusion of combined in vitro generated AD-MSC and bone marrow $(\mathrm{BM})$ derived HSC on day -14 . Bortezomib $1.3 \mathrm{mg} / \mathrm{m}^{2}$ with Methyl prednisone (MP) $125 \mathrm{mg}$ was administered intravenously on days $-12,-9,-6$ and -4 for conditioning. Transplantation was performed on day zero if 3 out of 4 tests were favorable, i.e. if anti-human globulin (AHG) test was $<20 \%$, DSA $<1000$ mean fluorescent intensity (MFI), FCM with T-cell was $<50$ and B-cell $<100$ median channel shift (MCS) and negative mixed lymphocyte reaction (MLR) performed on day -2. Additional conditioning included rabbit anti-thymocyte globulin (r-ATG) $1.5 \mathrm{mg} / \mathrm{kg} \mathrm{BW}$ given at the time of surgery (day 0 ) and Rituximab, 375 $\mathrm{mg} / \mathrm{m}^{2}$ given on day +1 . Bortezomib with MP was repeated after transplantation in the same doses described above, on days $+2,+5,+8$ and +11 and patient was discharged on day +15 after collecting samples for DSA and MLR.

TIP for group-2 was similar to that of group-1 except that no SCT was administered. Patients were administered DST intravenously instead of SCT on day -14 after the blood samples for negative cross-matching from donor and recipient were confirmed. Bortezomib with MP was administered on days $-12,-9$, $-6,-4$ followed by cross-matching on day -2 and transplantation was performed on day zero. Additional conditioning included rabbit anti-thymocyte globulin (r-ATG) $1.5 \mathrm{mg} /$ $\mathrm{kg}$ BW given at the time of surgery (day 0 ) and Rituximab $375 \mathrm{mg} / \mathrm{m}^{2}$ given on day +1 . Bortezomib with MP was repeated on days +2 , $+5,+8$ and +11 and patient was discharged on day +15 . Transplantation was performed if 3 out of 4 tests were favorable, i.e. if AHG was $<20 \%$, DSA $<1000 \mathrm{MFI}, \mathrm{T} / \mathrm{B}$ cell FCM was $<50 / 100$ MCS and MLR was negative.

\section{- Laboratory methodologies:}

\section{HLA typing, LCM, FCM and SA testing}

These were performed using conventional serological techniques defined by American Society of Histocompatibility and Immunogenetics (ASHI) (using one-Lambda pre-dot trays for HLA- A, B, DR), using auto cross-match, di-thiothretol and standard cytotoxicity method with mixed-cell population. $\mathrm{T}$ and B-lymphocytes were separately utilized for cross matching and donor-specific positivity was found with mixed-cell population. FCM was performed after enzyme (pronase) treatment to remove nonspecific binding proteins. Fluorescent isothiocynate (FITC) 
conjugated anti-CD3 (perCP) and anti-CD19 [phycoerythrin (PE)] monoclonal antibodies were used for labeling $\mathrm{T}$ and B-cells respectively. Analysis was carried out by gating 5000 to 10,000 lymphocytes. For DSA (IgG specific) HLA class-I and II screening was performed with SA Beads (One Lambda, CA) on Luminex platform following the manufacturer's protocol. Trimmed MFI values were obtained from the output file generated by the flow analyzer, and normalized using the formula: [(Sample \#N beads - Sample negative control (NC) beads) (Negative control serum \#N beads - Negative control serum NC beads)].

\section{- Procurement of stem cells:}

PBSC were collected from donors stimulated with granulocyte colony stimulating factor (Intas Ltd, India) $7.5 \mu \mathrm{g} / \mathrm{kg} \mathrm{BW}$ subcutaneously twice a day, for 2 days) (Cobe Spectra version 7-Gambro, China). Mean $176.7 \pm 22.63 \mathrm{ml}$ of PBSC with mean nucleated cell count of $3.33 \pm$ $0.07 \times 10^{5} \mathrm{cells} / \mu \mathrm{L}$ were collected and infused in peripheral circulation of patients on day -19 (with mean cell count of $14.47 \pm 2.07 \times 10^{8}$ cells/kg BW).

For HSC, $100 \mathrm{ml} \mathrm{BM}$ was aspirated from posterior superior iliac crest of donor under local anesthesia (LA) on day -22, after G-CSF stimulation for 2 days as described above. BM was subjected to in vitro culture and expansion using un-fractionated $\mathrm{BM}$ with stromal cells to increase the yield of CD34+ cells. Dulbecco's Modified Eagle's Medium (DMEM) with antibiotics, high glucose, essential amino acids, albumin and growth factors was used to generate HSC. Medium was replenished every other day for 9 days at the end of which supernatant was removed and cultured marrow was mixed with AD-MSC after testing for viability, sterility, staining and quantification.

For AD-MSC generation, about 10 grams adipose tissue was resected from anterior abdominal pad of fat of donor under LA after making a small incision on left lateral side below umbilicus. Sutures were taken after homeostasis was secured. This fat was collected in medium containing $\alpha$-MEM with albumin and antibiotics. Collagenase-I was added and incubated at $37^{\circ} \mathrm{C}$ for 1-hour on self-designed shaker at 35-40 RPM for digestion followed by low speed centrifugation. The supernatant and pellets were separately cultured in medium with same composition on $100 \mathrm{~cm}^{2}$ and $25 \mathrm{~cm}^{2}$ cell+culture dishes (Sarsted, USA) respectively, at $37^{\circ} \mathrm{C}$ with $5 \% \mathrm{CO}_{2}$ for 10 days. The medium was replenished every other day and harvested by trypsinization.

SC Testing Methodology and Release Criteria: Collected cells after being tested for sterility, viability and morphology, were subjected to flow cytometric analysis. Cells were mixed with cultured BM and infused in portal circulation of patients. Viability and sterility were checked by microscopy on modified Neubaeur's chamber after mixing with $1 \mathrm{~N}$ phosphate buffered saline and with trypan blue dye test. Small aliquot was also subjected to culture in Bactec 6050 [Becton Dickinson (BD), U.S.A.]. SCs were analysed using FACScan [BD, U.S.A.]. CD34+/ CD45+/CD33+ were counted using $\mathrm{CD} 33 \mathrm{mAb}$ (PE-conjugated), CD34 mAb (FITC-conjugated) and CD45 $\mathrm{mAb}$ (PerCP-conjugated) (BD Biosciences, U.S.A.). For AD-MSC, CD45-/90+ and CD73+, CD73 mAb (PE-conjugated), CD90 $\mathrm{mAb}$ (FITC-conjugated) were used.

\section{- SC administration:}

SC infusion in portal circulation was performed using 20 guaze needles under general anesthesia by securing omental vein via minilaparotomy approach. Totally $100 \mathrm{ml} \mathrm{BM}$ with mean nucleated cell count of $0.37 \pm 0.08 \times 10^{8} /$ $\mathrm{kg} \mathrm{BW}$ with mean CD34+ count of $21.8 \pm 16.3$ $\times 10^{4} / \mathrm{kg} \mathrm{BW}$ and $2 \mathrm{ml}$ of AD-MSC with mean nucleated cell count of $22.12 \pm 19.58 \times 10^{4} / \mu \mathrm{L}$ with CD $45-/ 90+/ 73+$ count of $19.4 \pm 8.9 \times 10^{4} /$ $\mathrm{kgBW}$ were mixed thoroughly and infused in portal circulation. FIGURE 2 depicts AD-MSC under microscope and in flow cytometry.

Group-2 donors received G-CSF $7.5 \mu \mathrm{g} /$ kgBW subcutaneously twice a day, for 2 days and then were bled to collect 1 unit of blood. The centrifuged $60 \mathrm{ml}$ blood was infused in to patients and remaining plasma and platelets were returned to the donors. Mean nucleated cell count of DST infused in peripheral circulation of group-2 patients was $46.2 \pm 17.3 \times 10^{3} / \mathrm{kg}$ BW with mean CD34+ count of $0.13 \pm 0.06 \times$ $10^{2} / \mathrm{kg} \mathrm{BW}$.

\section{- Post-transplant monitoring:}

Post-transplant monitoring for graft function and graft versus host disease (GVHD) was similar for both groups. This included skin morphology and excluding gastro-intestinal signs, complete blood counts, SCr, blood urea, electrolytes, urine routine and microscopy, liver function tests, stool for occult blood, patient weight, intake and output charts along with complete physical examination. Daily monitoring till 30 days post-transplant, alternate days for next 15 days, twice a week for next 1 month (till 3 months post-transplant), weekly till 6 months post-transplant, fortnightly till 7 th month and monthly thereafter for first year, every 2 months for second year, and every 3 months thereafter. Monitoring with ultrasonography and Doppler for graft assessment was performed weekly 

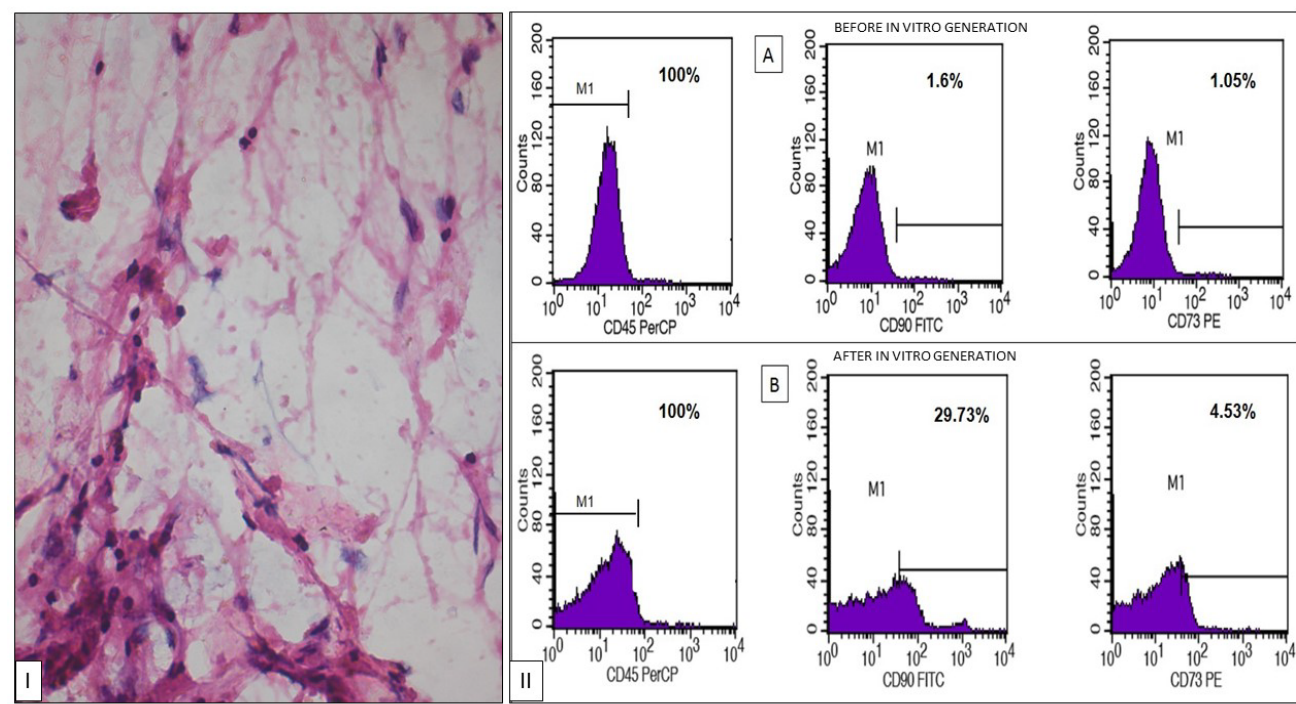

FIGURE 2: On left (I) is photomicrograph of in vitro generated adipose tissue derived mesenchymal stem (AD-MSC) cells, which appear in various stages of maturation ranging from round/ polygonal to elongated, fibroblastoid with centrally placed basophilic nuclei surrounded by eosinophilic granular cytoplasm, Hematoxylin and eosin stain, $x$ 200. On right (II) top is flow cytometry analysis of AD-MSC- before in vitro generation (out of $96.12 \%$ gated events, $0.19 \%$ are $C D 90+$ and $0.02 \%$ are CD $73+$ events) and below is after in vitro generation (increased to $31.66 \% \mathrm{CD} 90+$ and $2.64 \% \mathrm{CD} 73+$ events).

for 3 months post-transplant and then as per requirement. HIV, HBsAg, $\mathrm{HCV}$ monitoring was performed every 2 months for 1 st year and then as per requirement.

Protocol biopsies were performed after completion of 100 days of stable graft function and yearly in patients who gave informed written consent. Graft biopsy was also performed for dysfunction in all patients and managed as per standard guidelines. Rescue immunosuppression was started with rise of SCr or DSA $>5000$ MFI. Biopsy proven acute antibody (B-cell) mediated rejections (ABMR) were treated with one cycle of bortezomib, 3 plasmapheresis sessions (with $40 \mathrm{ml} / \mathrm{kg} \mathrm{BW} /$ session) and IVIg $5 \mathrm{gm}$. For T-cell mediated rejections (TCMR) not responding to $500 \mathrm{mg}$ MP for 3 days, r-ATG $1.5 \mathrm{mg} / \mathrm{kg} \mathrm{BW}$ was administered intravenously.

No conventional immunosuppression was administered to any patient. Posttransplant immunosuppression included monotherapy of Prednisone, $20 \mathrm{mg} /$ day orally for first month, $10 \mathrm{mg} /$ day in 2nd month and $5 \mathrm{mg} /$ day was continued thereafter. Rescue immunosuppression with Mycofenolate, 360 $\mathrm{mg}$ twice a day was started if the patients did not recover after anti-rejection therapy. CNI were added if mycofenolate was not effective. Repeat biopsy was planned if required and managed as per standard guidelines.

If Tacrolimus was started, levels were measured at weekly intervals for the first 2 months post- transplant, fortnightly for the next
2 months, and monthly thereafter using Siemens reagent flex kit (Siemens RxL Max) according to manufacturer's protocol maintaining trough levels of $4-7 \mathrm{ng} / \mathrm{mL}$. Cyclosporin $3 \mathrm{mg} / \mathrm{kg}$ $\mathrm{BW} /$ day was to be used if Tacrolimus was contraindicated. Trough levels were to be measured similar to that of Tacrolimus, with acceptable range of $70-150 \mathrm{ng} / \mathrm{ml}$. Sirolimus and Mycofenolate level measurement could not be performed due to un-availability of kits.

\section{Immune monitoring:}

Monitoring for DSA and MLR was performed every 3 months. MLR revealed absence of reactivity in all samples for 3 years and hence was discontinued subsequently. MLR of recipient and donor blood was carried out using anti-Bromodeoxyuridine (BrDU) (FITC) and 7-AAD (BD Biosciences, USA) according to manufacturer's protocol. Blood $(9 \mathrm{ml})$ of patient and donor was collected in sodium heparin vacuette. The diluted sample in normal saline was layered on concentration gradient separation medium (histoprep) and centrifuged at 1200 RPM for 12 minutes. The buffy coat was aspirated and taken in different centrifuge tubes. Cells were centrifuged again to remove the contaminating RBCs, etc. The prepared pellet was subjected to quantification following which $2 \times 10^{6}$ cells/well were loaded in to 6-welled plate. Half of the cell yield was subjected to irradiation (Cobalt irradiator, Bhabha Atomic Research Center, India) (7.5 cGY X 8 minutes). In total 4 wells were loaded 
for each test. In one well recipient's cells were loaded, in second, donor's cells were loaded, in 3rd well patient+donor cells were loaded and in 4th well patient+donor's irradiated cells were loaded. The plate was then incubated in $\mathrm{CO}_{2}$ incubator at $37^{\circ} \mathrm{C}$ overnight. After 18 hours of incubation BrdU was incorporated and 6 hours later, the cells were aspirated and subjected to flow cytometry analysis. The cell cycle positions and active DNA synthetic activities of cells were determined by analyzing the correlated expression of total DNA and incorporated BrdU levels. Apoptotic cells were defined as sub-G0/ $\mathrm{G} 1$, or resided in $\mathrm{G} 0 / \mathrm{G} 1, \mathrm{~S}$ or $\mathrm{G} 2+\mathrm{M}$ phases of the cell cycle that had recently synthesized DNA. The 7-AAD signal data was acquired in a linear mode.

\section{- Statistical analysis:}

Statistical analysis was performed using SPSS v 20 and Microsoft office 2007. Continuous data are presented as mean \pm standard deviation. Continuous variables were tested using independent student ' $\mathrm{t}$ ' test and Mann-Whitney 'U' test. Survival analysis was carried out using Kaplan-Meier method with log rank test; $\mathrm{p}<0.05$ was considered to be statistically significant.

\section{Results}

No infections or any other adverse side effects were observed due to TIP including conditioning or SCT/ DST in any patient of both groups. Patient survival was $100 \%$ for the first 2 years, $90 \%$ at 3 years and $80 \%$ at
5 years post-transplant in group-1 and 90\% for all 5 years post-transplant in group- 2 . Death-censored graft survival was $100 \%$ for first year in both groups, $90 \%$ at 5 years in group-1, and $70 \%$ at 5 years post-transplant in group-2. Kaplan Meier death-censored graft survival is displayed in FIGURE 3. One patient was lost to pneumonia at 33 months post-transplant and second patient was lost to chronic graft dysfunction with sudden cardiac arrest at home at 38 months post-transplant in group-1. One graft was lost at 17 months to chronic graft dysfunction with unexplained interstitial fibrosis, tubular atrophy (IF/TA) and absence of DSA. In group-2, 1 patient was lost to de novo focal segmental glomerulosclerosis associated complications at 10 months posttransplant. One graft was lost to CMV infection at 17 months, second graft due to IF/TA at 40 months and third graft to chronic TCR+ABMR at 60 months.

Two patients of group- 1 and 4 patients of group-2 did not give permission for protocol biopsy. All other patients of group-1 had normal biopsies till 4 years post-transplant (FIGURE 4) except 1 graft that revealed IF/ $\mathrm{TA}$ at 17 months. In group-2, 2 patients had normal biopsy till 2 years post- transplant, and 2 patients showed TCMR. Acute TCR+ABMR were noted in 2 patients of group-1, one at 33.4 months and second at 1.4 years post-transplant. Third patient developed acute TCMR at 155 days post-transplant and then subsequently IF/ TA at 1-year post transplant. In group-2, acute TCR+ABMR was observed in 1 patient at 70 days post-transplant. In second patient acute

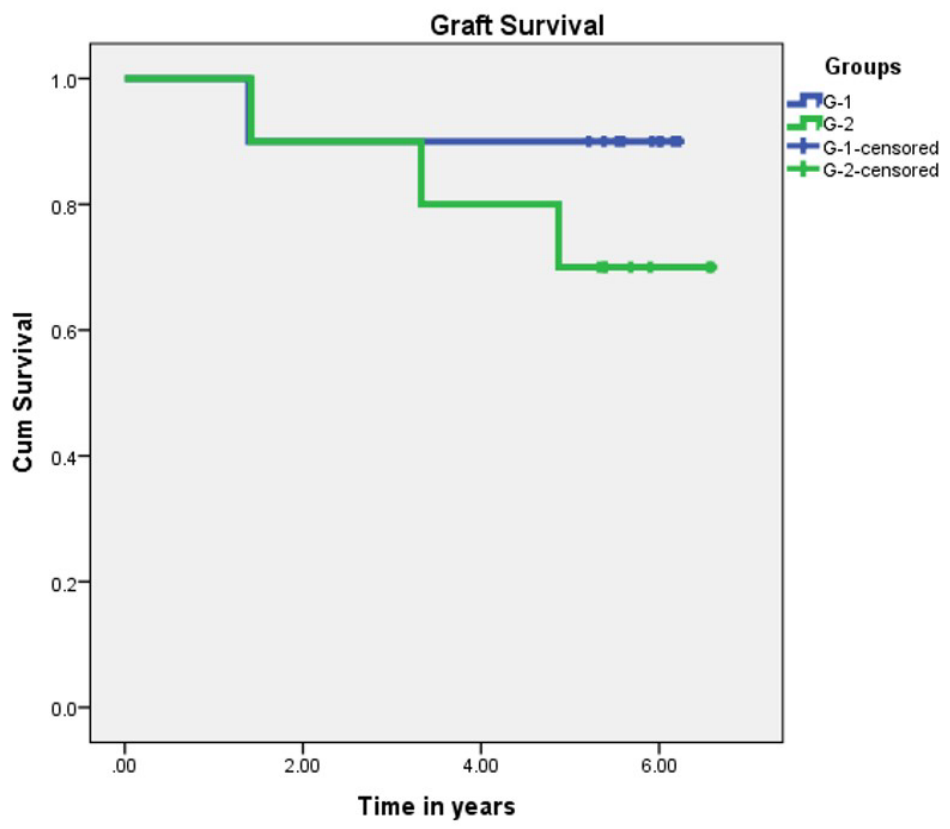

FIGURE 3: Death-censored renal allograft survival showing $90 \%$ survival at 5 years in group-1 (SCT) and $70 \%$ survival in group-2 (DST) depicted in Kaplan Meier analysis. 


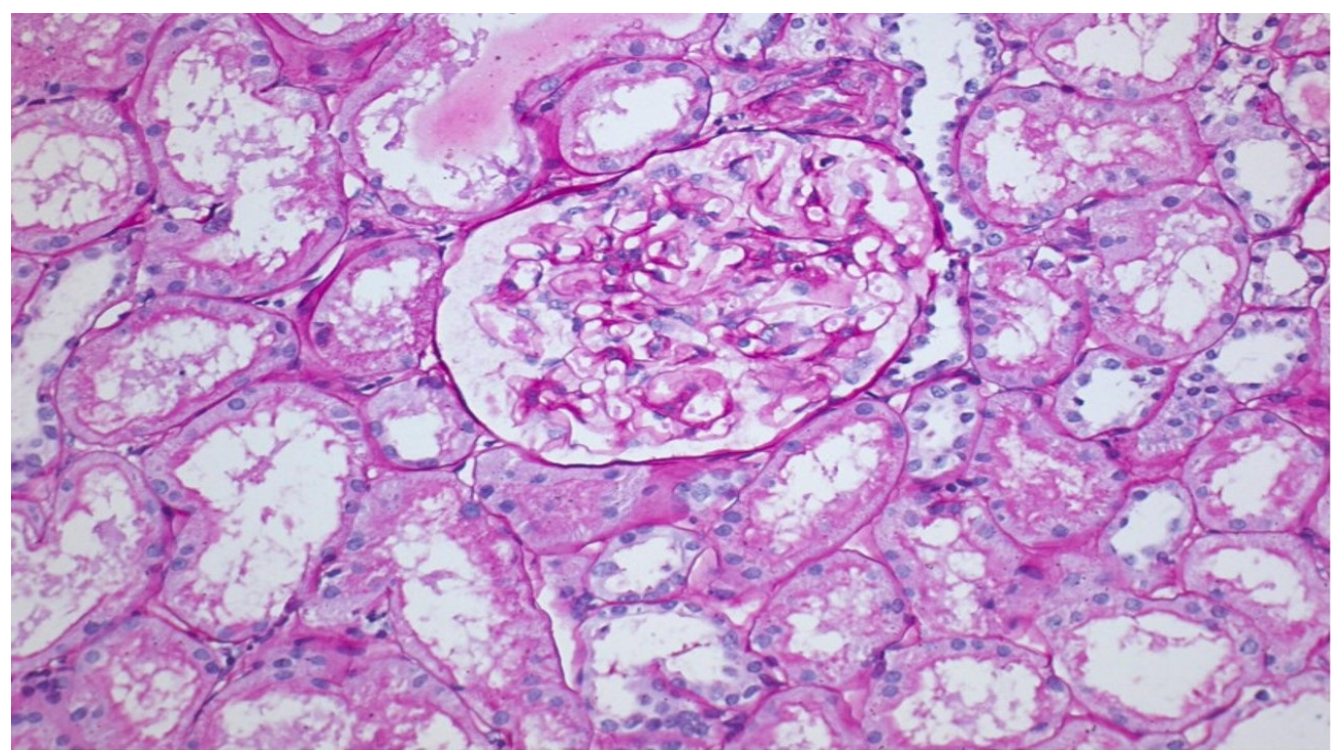

FIGURE 4: On left is normal protocol biopsy of a patient on no immunosuppression at 2 years posttransplant, showing one glomerulus with surrounding tubules, PAS stain, x 200.

TCMR was observed on 9 th post-transplant day followed by chronic active TCMR at 2.5 years post-transplant. Third patient developed chronic TCR+ABMR at 2.5 years post-transplant.

In group-1, over a mean follow-up of 5.43 \pm 0.37 years, out of 7 patients, 5 are on no conventional immunosuppression, and 2 are on mycofenolate, $360 \mathrm{mg} \mathrm{BD}+$ prednisone $5 \mathrm{mg} /$ day. In group- 2 , over a mean follow-up of $5.46 \pm 0.49$ years, out of 6 patients, 3 are on no conventional immunosuppression, 2 are on mycofenolate, 360 $\mathrm{mg} \mathrm{BD}$ and prednisone $5 \mathrm{mg} /$ day and 1 patient is on triple immunosuppression of Tacrolimus, $0.05 \mathrm{mg} / \mathrm{kg} \mathrm{BW} /$ day+Mycofenolate, $360 \mathrm{mg}$ $\mathrm{BD}$ +prednisone $5 \mathrm{mg} /$ day.

DSA (5000-6500 MFI, class $1 \pm 2$ ) appeared in 2 patients of group- 1 , at 4 months however graft biopsy and SCr remained unaffected. In group-2 persistence of DSA (5000- $6000 \mathrm{MFI}$, class $1 \pm 2$ ) was noted in 3 patients and was associated with deterioration of graft function.

Graft function in terms of SCr is depicted in FIGURE 5. Mean SCr of group-1 was $1.52 \pm$ $0.52 \mathrm{mg} / \mathrm{dL}$ vs. $1.97 \pm 1.18 \mathrm{mg} / \mathrm{dL}$ in group- 2 .

Thus group- 1 was better than group- 2 in terms of better SCr, lesser/ no immunosuppression requirement, lesser rejection episodes and normal protocol biopsies; however there was no statistically significant difference found between the two groups. MLR revealed absence of reactivity in all samples.

\section{Discussion}

MSC isolated from BM and described for the first time by Friedenstein et al, are multipotent SC derived from various sources [10]. They are negative for CD34/45/HLA-DR and express CD90/73/ 105 on their surface $[11,12]$. Treatment using MSCs was already approved for intractable GVHD following allogeneic SCT. More recently it has been suggested that the use of MSCs may play a role for temporary engraftment of BM for facilitation of induction of mixed chimerism to help accomplishing TI $[13,14]$. We learnt lessons from our experience of using HSCT for chimerism/ clonal deletion associated TI that some other pathway is also involved in TI. We also observed that there was no consistency and reproducibility of tolerance when we used HSC alone or with conditioning [15].

MSC can down-regulate GVHD and rejection $[16,17]$. Since these cells do not express HLA-DR, they can evade rejection initiated by T-cells. MSC were shown to convert antigen presenting cells into an inhibitory or suppressor phenotype via cell-to-cell contact, thus locking dendritic cells into a semi-mature state, thereby inducing peripheral tolerance associated with reduced IFN-F, IL-12, and TNF- $\alpha$ [18]. MSC are known to recruit and generate T-regs, and these T-regs induce infectious tolerance [19].

Scientists tested in vitro, the ability of BM derived MSC to induce proliferative response in allogeneic lymphocytes and potent T-cell mitogens [20]. They observed that MSCs failed to elicit a proliferative response. When MSCs were added to mixed lymphocyte reaction on day 0 and 3 , and also to mitogen-stimulated lymphocytes, there was more than $50 \%$ fall in proliferation. This response was also found to be dose dependent, higher the dose of MSCs, more the suppression of proliferation. However when 
Graft function in terms of $\mathrm{S}$. Creatinine (mg/dl) in group-1 (SCT) vs. group-2 (DST)

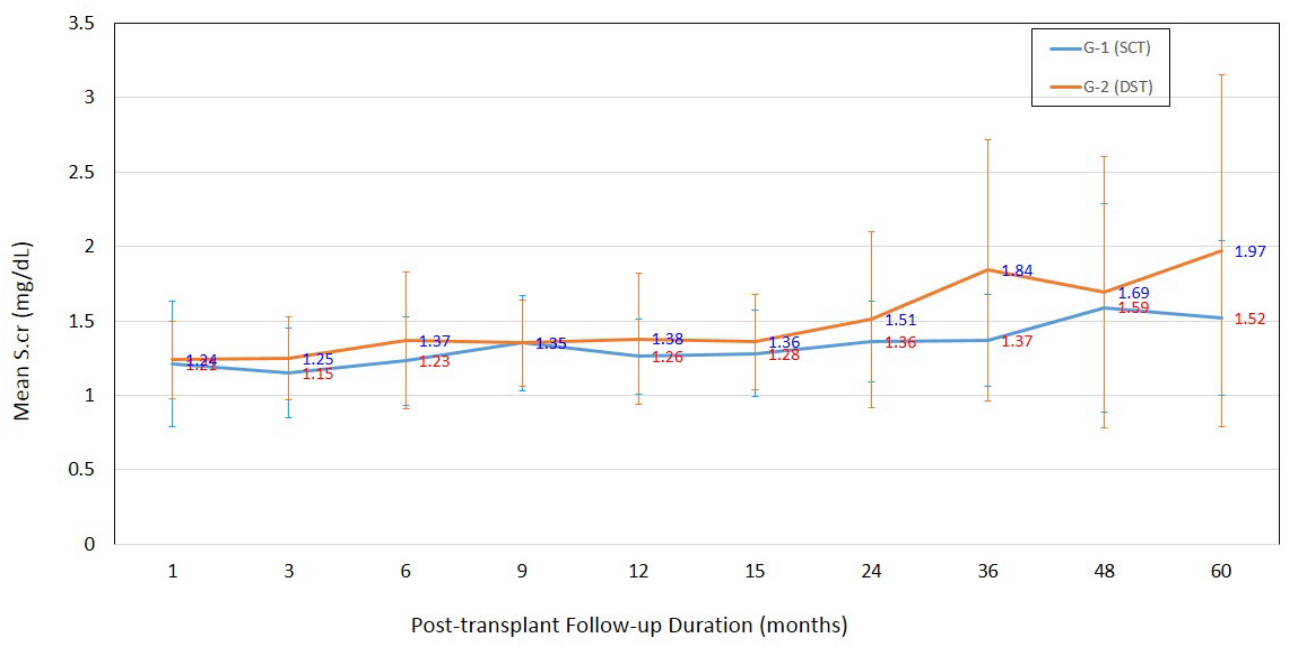

FIGURE 5: Mean serum creatinine ( $\mathrm{mg} / \mathrm{dL}$ ) of both groups over follow-up of 5 years.

IL-2 was added, the proliferation was increased. Then they injected intravenous donor MSCs in MHC-mismatched baboons. Subsequently they carried out autologous, donor and third party skin transplants. They observed that MSCs helped in prolonging the survival of skin grafts. Ge et al injected intravenously MSCs from wild type mice (WT-MSC) and indoleamine 2,3-dioxygenase (IDO) knock-out (IDO(-/) $\mathrm{C} 57 \mathrm{BL} / 6$ mice into BALB/c mice 24 hours after orthotopic renal transplantation from C57BL/6 mice.19 They observed that mice who were administered WT-MSCs achieved allograft tolerance. Graft histology was normal, there were no DSA, blood kynurenine levels were elevated and there was significantly high level of tolerogenic dendritic cells in addition to impaired CD4+T-cell responses. They also observed high frequency of CD4+CD25+ Foxp3+ Tregs in recipient spleens and donor grafts along with donor-specific CD25+cell depletion. This experiment established that MSCs recruit Tregs for TI. Casiraghi et al. carried out few experiments [21]. They injected single dose of MSC in portal vein of rat followed by heart transplant from semi-allogeneic donor after 7 days. In second experiment they carried out double infusion of MSCs on day -7 and in tail vein on day -1 pre-transplant. They observed that there was profound T-cell hypo-responsiveness in recipient along with prolonged cardiac allograft survival. However this pro-tolerogenic effect was abrogated with combined infusion of donor HSCs and MSCs. MSC induced tolerance was associated with expansion of donor-specific CD4+CD25+Foxp3+Tregs and impaired anti-donor Th1 activity. In addition, infusion of recipient-derived B6 MSCs tolerized semi-allogeneic cardiac allograft, but not a fully
MHC-mismatched BALB/c graft. They also observed that double intravenous pretransplant infusion of recipient-derived MSC had the same tolerogenic effect as the combined intraportal or intravenous MSC infusions. This experiment was encouraging for application in clinic. In this experiment they also established that single MSC infusion peri/ post-transplant were less effective.

We had started generating AD-MSC in our lab, hence we embarked on their use in LDRT to exploit their immunomodulatory and tolerogenic effect on the graft [9]. We have already succeeded in safe minimization of immunosuppression using SCT with adjuvant non-myeloablative conditioning [22-25].

The present study was carried out to find out the possibility of developing robust tolerance by peri-transplant immunosuppression using no long-term immunosuppressive treatment other than low dose Prednisone. We also wanted to find out whether AD-MSC can facilitate TI or whether DST alone can achieve TI. Our previous experience with HSC alone showed that in spite of achieving persistent mixed chimerism of about $1.8 \%$ at 18 months post-transplant, compared to $4 \%$ chimerism using combined $\mathrm{AD}-\mathrm{MSC}+\mathrm{HSC}$ in LDRT, the incidence of rejections was higher with HSC group [23]. This study confirmed with the reports that MSC were immunomodulatory and more tolerogenic than HSC $[8,16]$. We carried out SC infusion into portal circulation to facilitate grafting by taking the advantage of Kupffer cells and privileged tolerogenicity microenvironment of the liver [26].

Sporadic incidence of TI has been reported 
in the literature mainly as chance findings associated with patient non-compliance [27]. In a study of 120 living identical twin donor renal transplants in USA and 12 in the UK, graft survival was $88.96 \%$ in the US group and $75 \%$ in the UK group for 5 years and patient survival was $97.01 \%$ in the US group and $100 \%$ in the UK group during the same 5 -year follow-up period [28]. In a study on 12 patients who received HLA-matched renal allografts, under conditioning, success was reported in 8 patients at mean 25 months after transplantation [29]. However conditioning was used in all these studies on HLA identical donors and majority of patients were on some form of immunosuppression for the first 6 months or even longer. Attempts of TI using Alemtuzumab and Deoxyspergualin were tried in 13 patients. Unfortunately in mean 23 days, all patients had to be rescued with immunosuppression [30]. In another study on 22 HLA identical kidney transplants, patients received conditioning regimen of TLI and r-ATG followed by donor HSC [31]. Patients were initially maintained on Mycophenolate mofetil $(2 \mathrm{~g} /$ day $)$ and Cyclosporine for at least 6 months post-transplant and discontinued 6 to 17 months post-transplant. They reported success in TI associated with chimerism in 16 out of 22 patients for 5 years. This study also shows gradual withdrawal of immunosuppression after heavy conditioning and after using standard immunosuppression for almost 1 year. In another study, 15 HLA-mismatched LDRT recipients underwent low intensity conditioning with Fludarabine, Cyclophosphamide and total body irradiation, followed by transplantation and subsequent facilitator cell infusion on day +1 [32]. These patients were also maintained on immunosuppression consisting of Tacrolimus and Mycophenolate and weaned over one year. They reported success in majority of patients who had durable chimerism. All these studies are single-armed; patients were conditioned heavily and maintained on standard immunosuppression for a minimum of 6 months. As compared to them, the present study shows that over 5 years followup AD-MSC with HSC have proved superior to DST in sustaining TI. As compared to all other components, AD-MSC, HSC and nonmyeloablative conditioning have remained the cornerstone of success in TI in our experience. However we did not find any correlation of tolerance and chimerism [15,22-23,32-36]. Our 5-year follow-up in the present study shows total $70 \%$ graft+patient survival, with $50 \%$ on no conventional immunosuppression and 20\% on rescue immunosuppression in the stem cell arm versus total of $60 \%$ survival, with $30 \%$ survival on no immunosuppression and $30 \%$ on rescue immunosuppression in DST arm. UNOS/ European data of RT show 5 year graft survival ranging from $62 \%$ to $78 \%$ on standard triple immunosuppression [37]. The present study is of paramount importance for developing countries where patients succumb to infections due to triple immunosuppression after induction therapies. In addition, this success is across HLA barriers. The financial benefit of TIP using SCT in RT is translated in cost of $<\mathrm{US} \$ 10,000$ and post-transplant maintenance of $<$ US $\$ 100$ per month even in case of rescue immunosuppression. In addition, patients return to mainstream of life and join their families without incurring any financial or social burden and issue of non-compliance does not arise. In comparison, standard RT costs approximately US $\$ 30,000$ and maintenance immunosuppression is approximately US $\$ 500$ per month in addition to the expenses frequently needed for treatment of infections and other metabolic complications.

\section{- Limitations of the present study:}

The protocol using SCT appears lengthy and cumbersome as compared to the protocol using DST. Total hospital stay was about 41 days for protocol using SCT and 25 days for DST protocol. However the benefits coming out over the longer run make up for the long hospital stay when patient is inducted in protocol. Generation of AD-MSC requires expertise; hence it has not been easy to emulate this study. Hence DST protocol was experimented to find out whether it could be adopted by most of the centers. However with our experience we have found that DST can be replaced by PBSC and patients can be safely kept on minimum immunosuppression [15,22-24]. In addition monitoring is expensive. We could not perform cytotoxic $\mathrm{T}$ lymphocyte precursor analysis. We did not look for chimerism in the present study since we have observed that chimerism is not always associated with tolerance $[7,23]$. To our knowledge this is the first report documenting results of 50\% 5-year survival of patients and renal allografts on no conventional maintenance immunosuppressive treatment undergoing TIP using SCT under non-myeloablative conditioning of Bortezomib, Rituximab and r-ATG. This study opens new gateways for additional protocols using various forms of SCs for induction of tolerance in organ transplantation.

\section{Conclusion \\ SCT can facilitate transplant tolerance in LDRT following non-myeloablative} conditioning. 


\section{Declarations \\ - Ethical approval and consent to participate}

The study was approved by our institutional review board and study number was IKDRCITSCDB/MCDB-09-2009.

\section{Consent for publication}

Consent for publication was obtained from the all patients.

\section{Authors' contribution}

A V Vanikar: carried out all the lab and immunology work and wrote the manuscript and finalized it. The subject was $\mathrm{PhD}$ thesis work undertaken from 2009 to 20015 at King George's Medical University, Lucknow, India.

H L Trivedi: designed the protocol, was the treating Physician for all patients and edited the manuscript. He is the main $\mathrm{PhD}$ guide for this work.

\section{Acknowledgements}

We are grateful to all our patients and their donors who put their implicit trust in our work and volunteered to undergo the tolerance induction protocols as per our instructions. The corresponding author also conveys gratitude to $\mathrm{PhD}$ co-guides Prof. A. Kumar and Prof. S.C. Gopal for providing guidance and support in carrying out this research work and taking it to completion under the aeges of King George's Medical University, Lucknow, India. The members of our Institutional Review Board also receive our special thanks since they permitted this study. Our Physician colleagues, students, friends and all lab staff deserve special thanks. Without inputs, guidance, support and constant encouragement from all of them, this work would not have seen the light of this day.

\section{Institutional Clinical Trial No. and date}

IKDRCITS-CDB/MCDB-09-2009; dated 24th November 2008 (This work was $\mathrm{PhD}$ thesis subject in King George's Medical University, Lucknow, India- PhD enrollment No. KGPH100029, Sr. No. KGMU/PHD/ 10)

\section{Trial Registration number}

CTRI Registration reference number: REF/2017/02/013341 2017

Registered retrospectively on 8th February,

\section{Funding \\ None}

Consent of patients \& donors

Obtained

\section{Conflict of interest}

None of the authors declare any conflict of interest regarding the publication of this manuscript

\section{Funding agency \\ Nil}




\section{REFERENCES}

Billingham RE, Brent L, Medawar PB. Actively acquired tolerance of foreign cells. Nature 172,603-606 (1953).

Feng S, Ekong UD, Lobritto SJ, et al. Complete immunosuppression withdrawal and subsequent allograft function among pediatric recipients of parental living donor liver transplants. JAMA 307,283-293 (2012).

Levitsky J. Operational tolerance: past lessons and future prospects. Liver transplantation 17,222-232 (2011).

Ruiz P, Maldonado P, Hidalgo Y, et al. Transplant Tolerance: New Insights and Strategies for Long-Term Allograft Acceptance. Clinical and Developmental Immunology Article ID 210506, 15 pages (2013).

Auchincloss H. In search of the elusive Holy Grail: The mechanisms and prospects for achieving clinical transplantation tolerance. Am J Transplant 1,6-12 (2001).

T Fehr, Sykes M. Clinical experience with mixed chimerism to induce transplantation tolerance. Eur. Soc. For Organ Transplantation 21,11181135 (2008).

Morecki S, Leshem B, Eid A, et al. Alloantigen persistence in induction and maintenance of transplantation tolerance. J Exp Med 165,1468-1480 (1987).

Aggarwal S, Pittenger MF. Human mesenchymal stem cells modulate allogeneic immune cell responses. Blood 105(4),1815-1822 (2005).

Doshi GR, Mehta KM. Institute of Kidney Diseases and Research Centre and Dr. H.L. Trivedi Institute of Transplantation Sciences. A method of preparing mesenchymal stem cells from adipose tissue. Intellectual Property India. Granted on 5th November, 2015. Patent number 269775, 17th July, 2008.

Friedenstein AJP, Piatetzky-Shapiro II, Petrakova KV. Osteogenesis in transplants of bone marrow cells. J Embryol Exp Morphology 16, 381390 (1966).

Pittenger MF, Mackay AM, Beck SC, et al. Multilineage potential of adult human mesenchymal stem cells. Science 284,143-147 (1999).

Jiang Y, Jahagirdar BN, Reinhardt RL. Pluripotency of mesenchymal stem cells derived from adult marrow. Nature 418,41-49 (2002).

Reyes M, Verfaillie CM. Characterization of multipotent adult progenitor cells, a subpopulation of mesenchymal stem cells. Ann N Y Acad Sci 938,231-233 (2001).

Sykes M. Mixed chimerism and transplant tolerance. Immunity 14,417-424 (2001).

Vanikar AV, Goplani KR, Feroz A, et al. Operational tolerance in living-related renal transplantation: a single-center experience. Transplant Proc 43,15511558 (2011).

Ryan JM, Barry FP, Murphy JM, et al. Mesenchymal stem cells avoid allogeneic rejection. J Inflammation 2,8 (2005).

Oluwole OO, Depaz HA, Gopinathan $\mathrm{R}$, et al. Indirect allorecognition in acquired thymic tolerance induction of donor-specific permanent acceptance of rat islets by adoptive transfer of allopeptide-pulsed host myeloid and thymic dendritic cells. Diabetes 50,1546-1552 (2001).

Beyth S, Borovsky Z, Mevorach D, et al. Human mesenchymal stem cells alter antigen-presenting cell maturation and induce T-cell unresponsiveness. Blood 105,2214-2219 (2005).

Ge W, Jiang J, Arp J, et al. Regulatory T-cell generation and kidney allograft tolerance induced by mesenchymal stem cells associated with indoleamine 2,3-dioxygenase expression. Transplantation $\quad 90,1312-1320$ (2010)

Bartholomew A, Sturgeon C, Siatskas M, et al. Mesenchymal stem cells suppress lymphocyte proliferation in vitro and prolong skin graft survival in vivo. Exp Hematol 30,42-48 (2002).

Casiraghi F, Azzollini N, Cassis P, et al. Pretransplant infusion of mesenchymal stem cells prolongs the survival of a semiallogeneic heart transplant through the generation of regulatory T cells. J Immunol 181,3933-3946 (2008).

Trivedi HL, Vanikar AV, Modi PR, et al. Allogeneic hematopoietic stem cell transplantation, mixed chimerism and tolerance in living related donor renal allograft recipients. Transplant Proc 37,737-742 (2005).

Vanikar AV, Trivedi HL, Feroze A, et al. Effect of co-transplantation of mesenchymal stem cells and hematopoietic stem cells as compared to hematopoietic stem cell transplantation alone in renal transplantation to achieve donor hypo-responsiveness. Int Urol Nephrol 43,225-232 (2011).

Trivedi HL, Kaneku H, Terasaki PI, et al. Clonal deletion using total lymphoid irradiation with no maintenance immunosuppression in renal allograft recipients. Clin Transpl 265-280 (2009).

Trivedi HL, Terasaki PI, Feroz A, et al. Clonal deletion with bortezomib followed by low or no maintenance immunosuppression in renal allograft recipients. Transplantation 90,221222 (2010).

Gorczynski RM. Immunosuppression induced by hepatic portal venous immunization spares reactivity in IL-4 producing $\mathrm{T}$ lymphocytes. Immunol Lett 33,67-77 (1992).

Orlando G, Hematti P, Stratta RJ, et al. Clinical operational tolerance after renal transplantation: current status and future challenges. Ann Surg 252,915-928 (2010).

Kessaris N, Mukherjee D, Chandak P, et al. Renal transplantation in identical twins in United States and United 
Kingdom. Transplantation 86,15721527 (2008).

Scandling JD, Busque S, Shizuru JA, et al. Induced immune tolerance for kidney transplantation. N Engl J Med 365,1359-1360 (2011).

Kirk AD, Mannon RB, Kleiner DE, et al. Results from a Human Renal Allograft Tolerance Trial Evaluating T-Cell Depletion with Alemtuzumab Combined with Deoxyspergualin. Transplantation 80,1051-1059 (2005).

Leventhal J, Abecassis M, Miller J, et al. Tolerance Induction in HLA Disparate Living Donor Kidney Transplantation by Donor Stem Cell Infusion: durable chimerism predicts outcome. Transplantation 95,169-176 (2013).

Scandling JD, Busque S, Shizuru JA. Chimerism, graft survival, and withdrawal of immunosuppressive drugs in HLA matched and mismatched patients after living donor kidney and hematopoietic cell transplantation. Am J Transplant 15,695-704 (2015).

Vanikar AV, Trivedi HL, Kumar A, et al. Mesenchymal stem cells and transplant tolerance. Nephrology (Carlton) 19,369-374 (2014).

Vanikar AV, Trivedi HL. Stem Cell
Transplantation in Living Donor Renal Transplantation for Minimization of Immunosuppression. Transplantation 94,845-850 (2012).

Trivedi HL, Vanikar AV. Mesenchymal stem cells and solid organ transplantation. CellR4 1,123-136 (2013).

Vanikar AV. Transplantation tolerance; myth or reality? Journal of Nephropathology 3,18-21 (2014).

Gondos A, Döhler B, Brenner H, Opelz G. Kidney graft survival in Europe and the United States: strikingly different long-term outcomes. Transplantation 95,267-274 (2013). 